\title{
Non-rheumatic mitral incompetence and papillary muscle dysfunction
}

\author{
Max ZoOB \\ M.D., M.R.C.P. \\ Consultant Cardiologist, Regional Cardiac Unit, \\ Brook Hospital, London, S.E. 18
}

\begin{abstract}
Summary
Non-rheumatic mitral incompetence may be caused by a variety of conditions affecting the relationships between the papillary muscles, chordae tendineae and mitral valve cusps. Among the commonest of these conditions are ventricular dilatation, ischaemia, congenital and familial abnormalities, senile atrophy of the muscles and spontaneous rupture. There is a very wide range of severity of the resultant clinical syndromes. Some patients are asymptomatic, others are moderately or severely incapacitated, while the severest cases may have acute pulmonary oedema which may be rapidly fatal. The diagnosis is strongly suggested by a late systolic murmur which may be initiated by a mid-systolic click while in others there is the unusual combination of an ejection-type systolic murmur with a quick-rising pulse. The diagnosis can be conclusively established by cineangiography and severe cases can be greatly helped by replacement of the mitral valve with a prosthesis.
\end{abstract}

\section{Introduction}

In recent years it has been realized that abnormalities of structure or function of the papillary muscles and chordae tendineae can lead to important disturbances of mitral valve function (Sanders et al., 1965; Barlow \& Bosman, 1966; Linhart \& Taylor, 1966; Raftery, Oakley \& Goodwin, 1966; Roberts, Braunwald \& Morrow, 1966; Stannard et al., 1967; Burch, de Pasquale \& Phillips, 1968). The term papillary muscle dysfunction has been used to cover the wide variety of clinical syndromes which may result. It is, however, far from satisfactory because the function of the chordae cannot be dissociated from that of the papillary muscles and the function of both of these structures has to be considered in relation to the valve cusps. For example chordae which are too long for normal valve cusps may allow incompetence. but chordae which are of normal length may permit incompetence if the cusps are unduly voluminous. It is quite clear, therefore, that the valve mechanism consists of three inseparable components, the papillary muscles, chordae and cusps. It is quite inappropriate to speak of a subvalvar mechanism of mitral incompetence and the term 'sub-valvar mitral incompetence' is even worse for it suggests that regurgitation is occurring from ventricle to atrium through some orifice below the valve. 'Non-rheumatic mitral incompetence', though clumsy, appears to be the only possible alternative but since the term papillary muscle dysfunction has obtained fairly widespread acceptance it is used as an alternative in the sense indicated in this introduction.

The normal function of papillary muscles and chordae

These structures have a crucial function to perform in maintaining a suitable position of the valve cusps throughout ventricular systole. During the isometric phase of ventricular function sufficient tension must be generated in the papillary muscles to prevent prolapse of the cusp upward into the atrium but not so much to pull them down into the ventricle and open the valve. Then as the ventricular cavity shortens during the ejection phase the papillary muscles must shorten correspondingly to take up the slack in the chordae and prevent prolapse and regurgitation.

Situated as they are directly under the endocardium, the papillary muscles are as remote as is possible from the coronary blood supply. They are, therefore, particularly susceptible to ischaemia. Normally the antero-lateral papillary muscle derives its blood supply from the circumflex branch of the left coronary artery but sometimes there is an accessory supply from the anterior descending branch of the left coronary artery. The posteromedial muscle is supplied either from the circumflex branch of the left coronary artery or the posterior descending branch of the right coronary. 


\section{Aetiology and pathology}

The main causes of non-rheumatic mitral incompetence and papillary muscle dysfunction are shown in Table 1. Dilatation of the left ventricular cavity alters the line of pull of the muscles which then tend

TABLE 1. Causes of non-rheumatic mitral incompetence and papillary muscle dysfunction

1. Ventricular dilatation

Aortic incompetence

Left ventricular failure

2. Ischaemia

Localized, of papillary muscle

Infarct \pm aneurysm

Fibrosis

3. Atrophy

Senile

Cachexia

4. Congenital anomalies

5. Rupture

6. Endocardial disease

Endocarditis

Fibro-elastosis

Endomyocardial fibrosis

7. Myocardial disease

Infiltration

Cardiomyopathy

to hold the valve open during ventricular systole. Such dilatation is particularly prone to occur in aortic incompetence and the secondary mitral incompetence which develops in this way may be so great as to dominate the clinical picture. Any other cause of left ventricular failure and dilatation, however, will act in the same way. The papillary muscles may be affected selectively by ischaemia or as part of a cardiac infarction or ventricular aneurysm. Atrophic shrinkage of the papillary muscles is common in the elderly who often have a noisy murmur but only slight mitral incompetence. Atrophy also occurs in cachectic conditions. Congenital abnormalities are probably fairly common but the lack of morbid anatomical studies makes it difficult to be sure just how often these are responsible for mild degrees of mitral incompetence. Certainly, however, this can be due to abnormalities of length and origin of the chordae or muscles. In some cases the abnormalities are familial and the clinical picture is then fairly characteristic and is described below. Finally, dysfunction of the mechanism may be caused by rupture of the muscles or chordae either spontaneously or following bacterial endocarditis. The remaining possibilities shown in Table 1 are rather uncomm on.

\section{Haemodynamics}

In mild cases the haemodynamics are little altered and may indeed be entirely normal. The acute development of mitral incompetence, however, causes an extreme elevation of left atrial pressure and pulmonary venous pressure leading to pulmonary oedema. Pulmonary vaso-constriction may result with extreme pulmonary hypertension. The pulmonary artery pressure in such cases is usually well over $60 \mathrm{mmHg}$ and the pulmonary vascular resistance considerably over 9 units (normal 1-3). In such cases there is both left and right ventricular hypertrophy.

\section{Clinical features}

A wide range of clinical syndromes occurs in this disorder, separable with some overlapping into five main groups.

\section{Asymptomatic non-familial cases}

These patients are discovered on routine examination to have a late systolic murmur as an isolated abnormality (Fig. 1). There are no relevant symptoms. X-ray of the chest is normal and there is no enlargement of the left atrium. Proof of the diagnosis

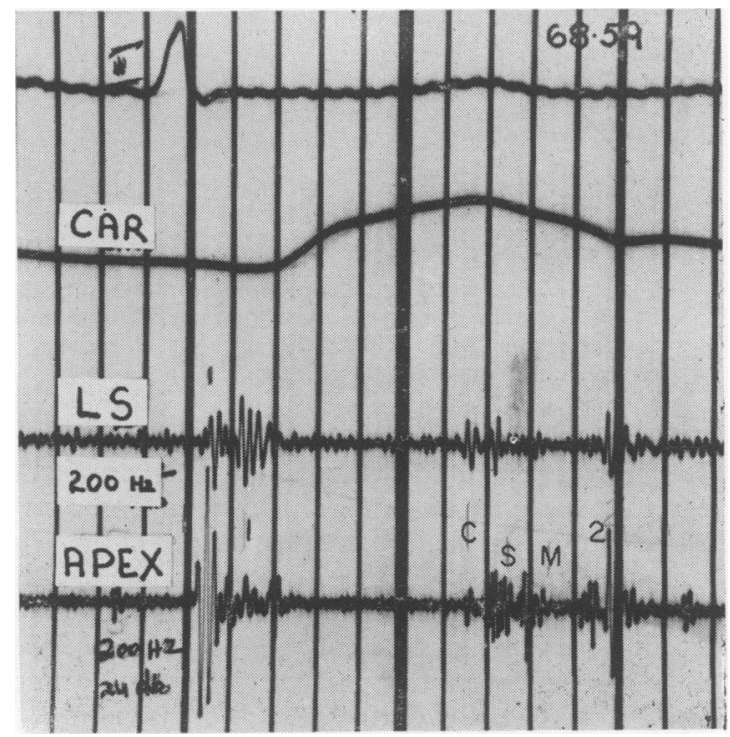

FIG. 1. A phonocardiogram showing a late systolic murmur (SM) following a mid-systolic click (C). These are characteristic findings in Groups 1, 2 and 5.

has been obtained in some cases by cineangiography which shows slight mitral incompetence confined to the latter half of systole. A few cases in this group show flattened or inverted $T$ waves in leads 2, 3 and aVF and this links them with the next group.

2. Ischaemic fibrosis or infarction confined to the papillary muscles

These patients do not invariably give a history of ischaemic cardiac pain and when they do, it sometimes has atypical features. Occasionally, however, 


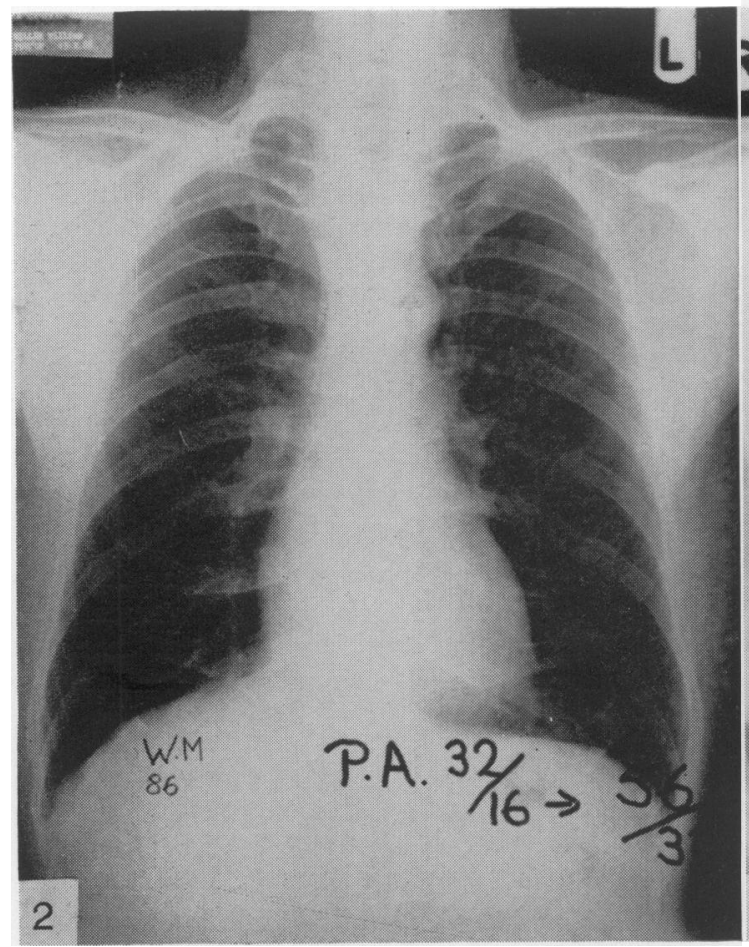

Fig. 2. The chest X-ray of a patient in Group 2. The heart is not enlarged and the pulmonary artery pressure of $32 / 16 \mathrm{mmHg}$ at rest was nearly normal. However, it rose abnormally to $56 / 30 \mathrm{mmHg}$ on effort, indicating a raised pulmonary vascular resistance.

the patient will describe typical central chest pain lasting for $\frac{1}{2} \mathrm{hr}$ or so without, however, any of the severe symptoms of cardiac infarction. Sometimes there is transient slight breathlessness on effort. The only abnormal sign in these cases is a late systolic murmur (Fig. 1). It seems that the infarcted or fibrotic papillary muscle can hold the mitral valve closed during the early part of systole but cannot shorten sufficiently during the ejection period to prevent regurgitation in the latter half of systole.

$X$-ray of the chest may show a normal sized heart or there may be a slight cardiac enlargement (Figs. 2 and 3). The cardiogram often shows flattening or inversion of the $T$ waves in leads 2,3 and $\mathrm{aVF}$ sometimes also in V5 and 6 (Fig. 4).

\section{Cardiac infarction involving the papillary muscles (sometimes with ventricular aneurysm)}

These patients may present with a typical history of cardiac infarction. This, however, is not invariable and sometimes the pain is atypical or even absent. In such cases the patient may present with acute breathlessness due to pulmonary oedema or breathlessness on effort. There is severe interference

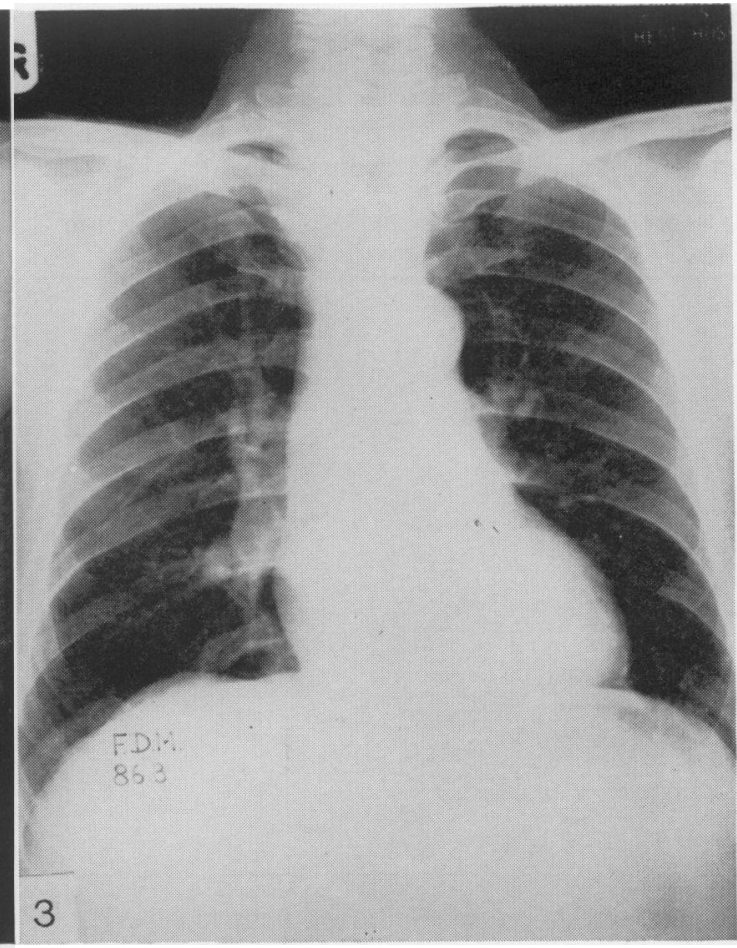

Fig. 3. The chest X-ray of a patient in Group 2. The heart is considerably enlarged and appreciable pulmonary hypertension due to mitral disease is indicated by the enlarged main pulmonary arteries with attenuated lower lobe branches and enlarged upper lobe veins.

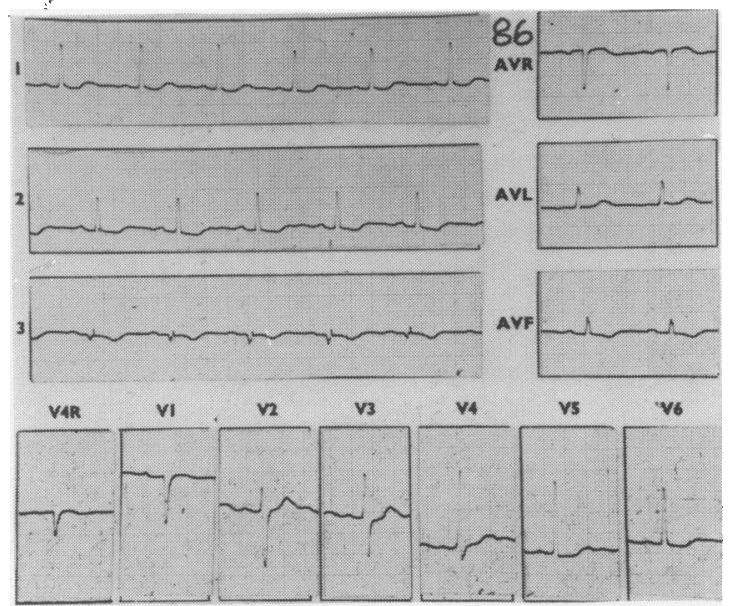

Fig. 4. A cardiographic pattern often seen in Groups 1, 2 and 5 . The $T$ waves are inverted (or flat) in leads 2,3 , aVF, V5 and V6. 
with mitral valve function and usually the infarcted papillary muscle fails to hold the valve shut throughout systole so that a pansystolic murmur results. The patient presents signs of severe acute mitral incompetence with high left atrial pressures, pulmonary oedema and pulmonary hypertension. There is a small volume, sharply rising pulse. The jugular venous pressure is considerably raised and ' $a$ ' waves are prominent. Sinus rhythm is the rule. There is evidence of over-activity of both ventricles and commonly there is tachycardia with gallop rhythm and some peripheral oedema. There may be rales over the lungs.

X-ray of the chest shows considerable cardiac enlargement involving both right- and left-sided chambers. The pulmonary artery is enlarged as are its main branches but the peripheral branches may be attenuated (Fig. 5). The left atrium is not enlarged.

The cardiogram in this particular case did not show a typical cardiac infarction pattern but only

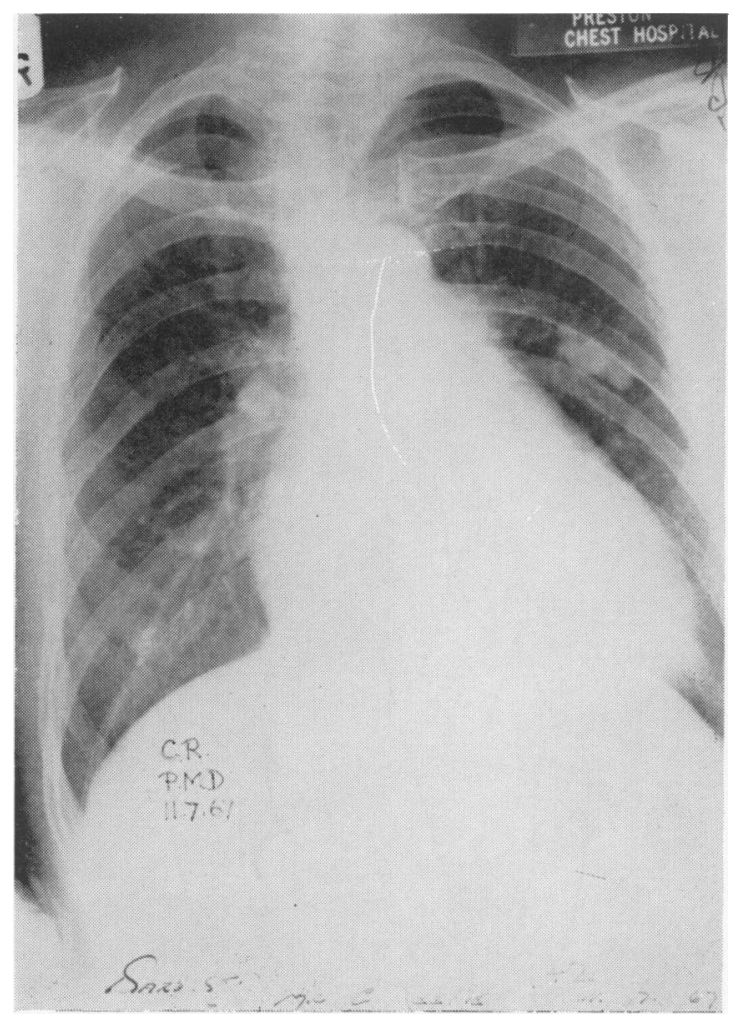

Fig. 5. X-ray of a patient with an extensive anterior cardiac infarction and ventricular aneurysm involving the papillary muscles. There is gross cardiac enlargement and the large main pulmonary arteries and attenuated lower lobe branches indicate pulmonary hypertension due to mitral disease. abnormal left axis deviation with S-T depression. However, the diagnosis was confirmed at operation at which the mitral valve was replaced with a prosthesis.

\section{Rupture of chordae tendineae}

Rupture of the chordae may occur spontaneously in apparently normal subjects or in patients known to have a systolic murmur presumably indicating congenital mitral valve abnormality. It may also complicate subacute bacterial endocarditis. In some patients there is a history of sudden severe chest pain on unaccustomed effort. In others, however, there is no such history and the presentation is with sudden breathlessness on effort or in bed at night (Fig. 6). Alternatively they may present with congestive heart failure and peripheral oedema. The physical signs resemble those of Group 3 but the murmur is not always pansystolic. The timing depends not only upon the function of the papillary muscles and chordae but also upon the degree of ventricular dilatation. Quite often regurgitation is confined to mid-systole and the murmur is maximal at that time resembling an ejection type murmur (Fig. 7). Moreover, if the posterior cusp prolapses the regurgitant jet may be directed over the anterior cusp towards the root of the aorta so that the murmur radiates to the base of the heart and even into the neck completing the resemblance to the murmur of aortic stenosis (Fig. 8). There is, however, little difficulty in differentiating this condition for in rupture of the chordae the ejection-type murmur is accompanied by a small-volume, sharply rising arterial pulse instead of the anacrotic pulse of aortic stenosis. If the patient survives, signs of pulmonary hypertension develop. It is noteworthy that the left atrium is not enlarged (Fig. 9).

\section{Congenital and familial abnormalities of the chordae tendineae}

A few of these patients rather surprisingly present with chest pain which, however, does not usually have the typical characteristics of ischaemic cardiac pain. It may be felt in the centre of the chest but may be stabbing in quality and unrelated to effort. In many patients, however, there are no symptoms whatsoever and the murmur is discovered on routine examination. As a rule there is but one physical sign consisting of a mid-systolic click followed by a murmur which continues to the second heart sound (Fig. 1). The cardiogram is usually normal but occasionally shows the pattern described in Groups 1 and 2 (Fig. 4). X-ray of the chest shows no abnormalities but in patients submitted to cineangiography prolapse of the posterior cusp of the mitral valve with late systolic mitral regurgitation, has been 


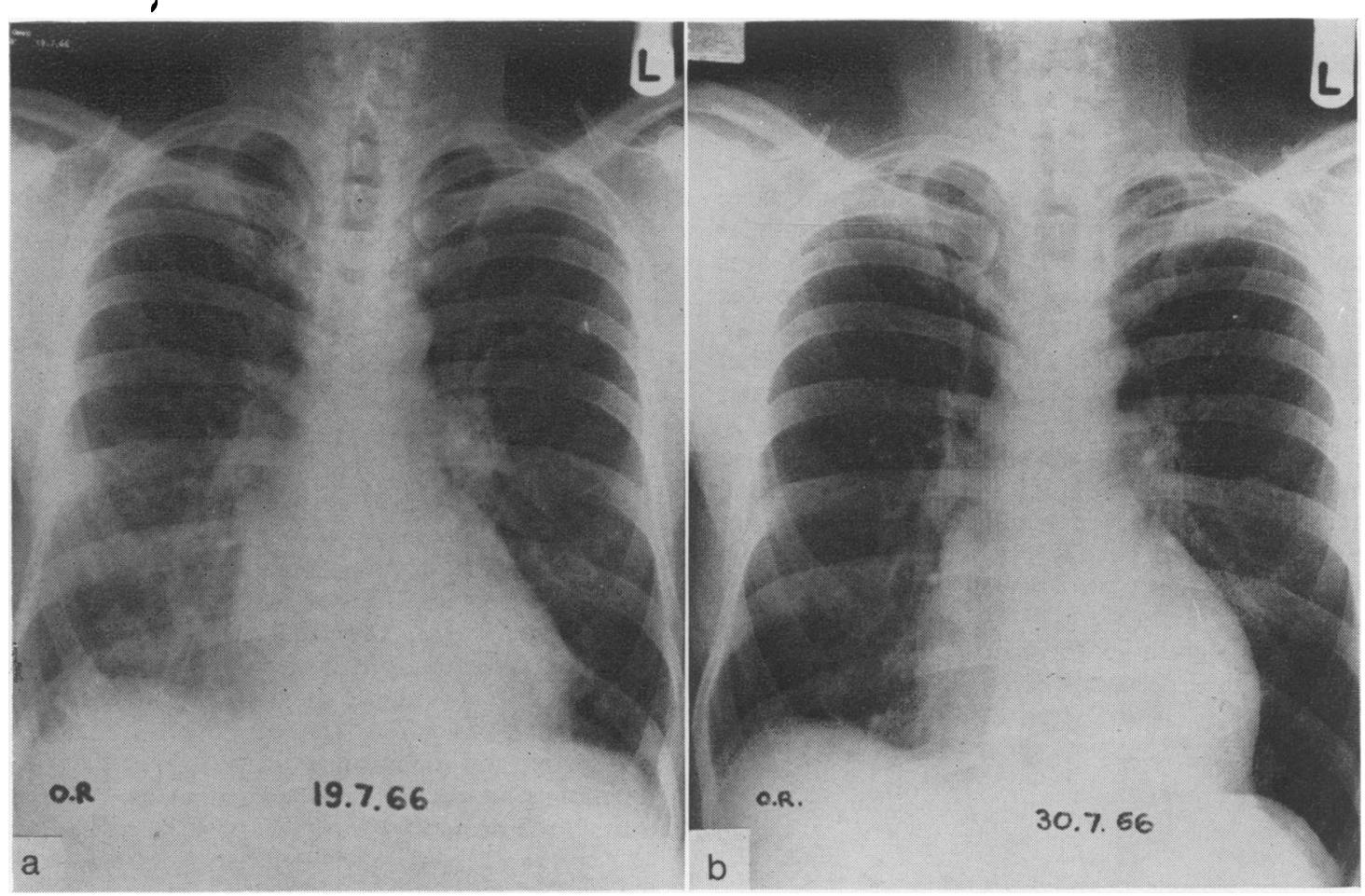

FIG. 6. Chest X-rays from a patient with ruptured chordae tendineae who presented with paroxysmal nocturnal dyspnoea. (a) There is pulmonary oedema particularly involving the right middle and lower zones. (b) Following intensive medical treatment, the pulmonary oedema has resolved but there is some cardiac enlargement and evidence of pulmonary hypertension.

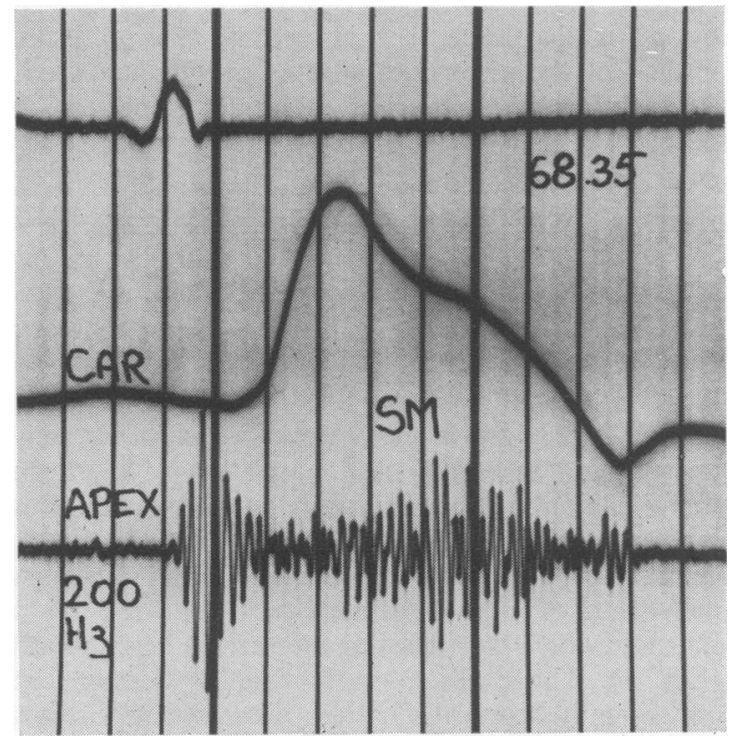

Fig. 7. The phonocardiogram from the patient with ruptured chordae tendineae shown in Fig. 6. The systolic murmur (SM) is maximal in mid-systole and resembles an ejection murmur (see text).

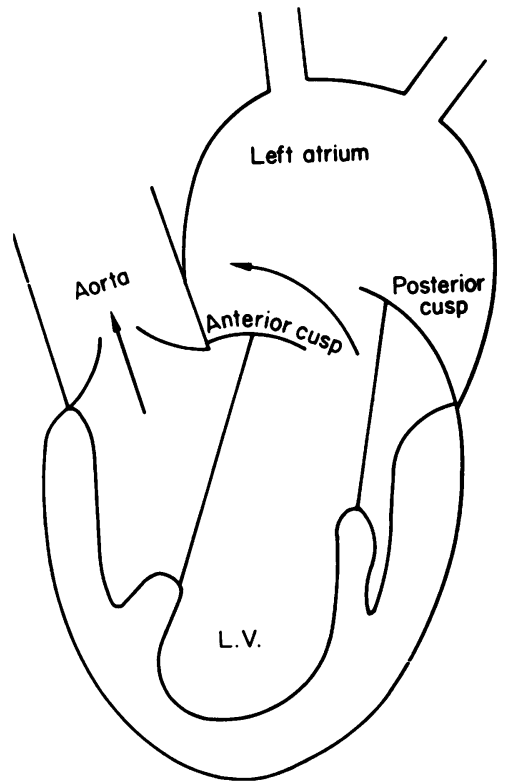

Fig. 8. Prolapse of the posterior cusp may allow the regurgitant jet to impinge on the root of the aorta. The murmur is then transmitted to the base of the heart and even to the neck (after Burch et al., 1968). 


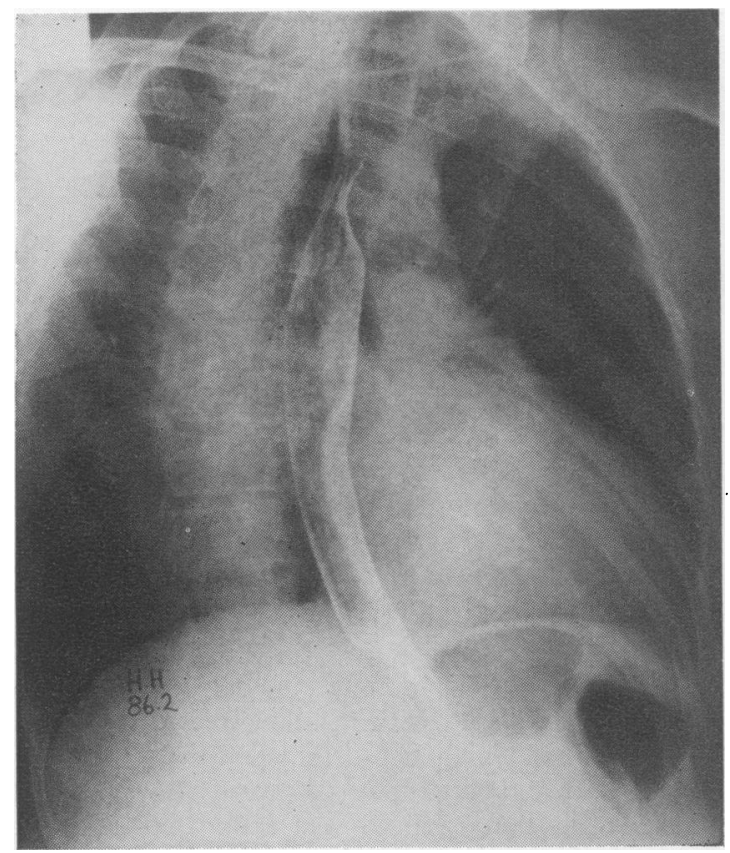

Fig. 9. Right oblique X-ray with barium swallow from a patient with ruptured chordae. As usual in nonrheumatic mitral incompetence the left atrium is not enlarged. This is probably because in Groups 1 and 5 the condition is insufficiently severe and in the others it is of short duration and the left atrium is uncompliant.

demonstrated. Some of these patients develop subacute bacterial endocarditis and Barlow \& Bosman (1966) have pointed out that sudden death occurred unexpectedly frequently in their large series of cases of this kind.

\section{Differential diagnosis}

In cases with a late systolic murmur, whether or not this is preceded by a mid-systolic click, the diagnosis presents little difficulty although it may be hard to be sure whether the aetiology is congenital or ischaemic. In patients with an ejection-type systolic murmur differentiation from aortic stenosis is easy on the basis of the character of the arterial pulse as already mentioned. There may, however, be some difficulty in differentiating an obstructive cardiomyopathy. In this condition, there is usually some mitral incompetence which may in fact be due to papillary muscle dysfunction. A similar mid-systolic murmur with quick-rising small volume pulse is found, but a fourth heart sound is common rather than the third sound found in ruptured chordae or primary papillary muscle dysfunction. Amyl nitrate inhalation intensifies the murmur of obstructive cardiomyopathy by diminishing the ventricular diastolic volume and therefore increasing the obstruction. By contrast it softens the murmur of other types of mitral incompetence by reducing the peripheral resistance and so encouraging forward flow.

Cineangiography is the most reliable method of establishing the diagnosis for it is usually possible to demonstrate prolapse of a cusp if this is the correct diagnosis. Moreover, it will then be seen that the ventricular cavity is large and not small and deformed as in the obstructive cardiomyopathies. Gradients in the outflow tract of the ventricle are much less dependable signs because they may be detected in ventricular hypertrophy of any cause and can easily be simulated by artefacts. Some patients in whom the disorder is due to cardiac infarction may present difficulty in diagnosis if the cardiogram does not conclusively reveal this. These patients would then present as cases of unexplained mitral incompetence presumably of rheumatic origin. In male subjects, however, this would be expected to be accompanied by severe calcification of the valve which would at once suggest the correct diagnosis. There would also be considerable left atrial enlargement and atrial fibrillation would be expected.

\section{Prognosis}

This largely depends upon the severity of the mitral incompetence. If this is mild the patient has a nearly normal expectation of life but is liable to the risk of subacute bacterial endocarditis and a few patients die suddenly and inexplicably.

Acute mitral incompetence due to papillary mitral dysfunction can be rapidly fatal, but many patients respond remarkably to medical treatment with digoxin and diuretics (Fig. 6). If they respond only partially or not at all, they can be dramatically helped by replacement of their mitral valve with a prosthesis.

\section{References}

BARlow, J.B. \& Bosman, C.K. (1966) Aneurysmal protrusion of the posterior leaflet of the mitral valve. Amer. Heart $J$. 71, 166.

Burch, G.E., De Pasquale, N.P. \& Phillips, J.H. (1968) The syndrome of papillary muscle dysfunction. Amer. Heart J. 75, 399.

LINHART, J.W. \& TAYLOR, W.J. (1966) The late apical systolic murmur. Amer. J. Cardiol. 18, 164.

RafTery, E.B., OAKLeY, C.M. \& Goodwin, J.F. (1966) Acute subvalvar mitral incompetence. Lancet, ii, 360.

RoberTs, W.C., Braunwald, E. \& Morrow, A.G. (1966) Acute severe mitral regurgitation secondary to ruptured chordae tendineae. Circulation, 33, 58.

Sanders, C.A., Scannell, J.G., Harthorne, J.W. \& AUSTEN, W.G. (1965) Severe mitral regurgitation secondary to ruptured chordae tendineae. Circulation, 31, 506.

Stannard, M., Sloman, J.G., Hare, W.S.C. \& Goble, A.J. (1967) Prolapse of the posterior leaflet of the mitral valve. Brit. med. J. 3, 71 . 